\title{
Tromboembolismo Venoso em Idade Pediátrica: Estudo Retrospetivo de 15 Anos
}

\section{Venous Thromboembolism in Pediatric Age: A 15 Year Retrospective Review}

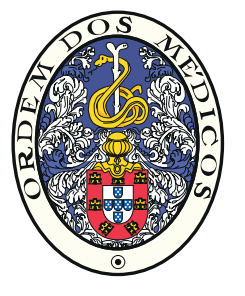

\author{
Joana Verdelho ANDRADEه ${ }^{1}$, Joana MAGALHÃES ${ }^{1}$, Catarina RESENDE¹, Dora GOMES ${ }^{1}$, Gabriela LARANJO', \\ Joana CAMPOS ${ }^{1}$, Elisabete SANTOS ${ }^{1}$, Cristina FARIA ${ }^{1}$ \\ Acta Med Port 2018 Sep;31(9):489-495 - https://doi.org/10.20344/amp.9639
}

\section{RESUMO}

Introdução: O tromboembolismo pulmonar e trombose venosa profunda ocorrem em idade pediátrica com incidência, morbilidade e mortalidade desconhecidas. O objetivo foi rever epidemiologia, apresentação clínica, exames complementares de diagnóstico e prognóstico de doentes com tromboembolismo pulmonar e trombose venosa profunda.

Material e Métodos: Estudo retrospetivo, descritivo e analítico de doentes pediátricos internados num hospital de nível II por tromboembolismo pulmonar e trombose venosa profunda, entre 2000 e 2014. Estudaram-se características demográficas, história clínica, comorbilidades e fatores de risco.

Resultados: Foram internados 11 doentes (sete com tromboembolismo pulmonar, cinco com trombose venosa profunda e um com ambos), $64 \%$ do género feminino e idade média de 16 anos. Todos os doentes com tromboembolismo pulmonar referiam toracalgia/ dispneia, $25 \%$ síncope/palpitações e $25 \%$ febre. Todos os doentes com trombose venosa profunda referiam dor no local da obstrução, $83 \%$ edema/cianose do membro afetado e $17 \%$ febre. O estudo da trombofilia positivo foi o fator de risco mais frequente nas duas entidades. O valor médio dos D-dímeros foi $3252 \mathrm{ug} / \mathrm{L}$ e $2660 \mathrm{ug} / \mathrm{L}$ no tromboembolismo pulmonar e trombose venosa profunda, respetivamente. Todos os doentes iniciaram anticoagulação, três necessitaram de cuidados intensivos, três apresentaram sequelas e houve um óbito.

Discussão: Todos os doentes tinham pelo menos um fator de risco associado e as condições de hipercoagulabilidade herdadas foram o fator de risco mais frequentemente encontrado nos nossos adolescentes.

Conclusão: $O$ aumento da incidência na população pediátrica descrito na literatura pode ser atribuído à crescente sensibilização para esta patologia, aos avanços médicos e aumento da sobrevida de doenças crónicas. Escasseiam recomendações baseadas na evidência que identifiquem os doentes com risco de trombose, para que as decisões possam ser tomadas de forma cuidadosa, equilibrando o risco e benefício em cada caso.

Palavras-chave: Criança; Embolia Pulmonar; Trombose Venosa

\section{ABSTRACT}

Introduction: Pulmonary thromboembolism and deep venous thrombosis occur in pediatric age, with unknown incidence, morbidity and mortality. Our aim is to review the epidemiology, clinical presentation, complementary diagnostic tests and prognosis of patients with pulmonary thromboembolism and deep venous thrombosis.

Material and Methods: Retrospective, descriptive and analytical study of pediatric patients admitted to a Level II hospital for pulmonary thromboembolism and deep venous thrombosis, between 2000 and 2014. Demographic characteristics, clinical history, comorbidities and risk factors were studied.

Results: Eleven patients ( $n=7$ pulmonary thromboembolism, $n=5$ deep venous thrombosis, $n=1$ both), $64 \%$ females and with 16 years old average, were admitted. All patients with pulmonary thromboembolism presented symptoms of chest pain and/or dyspnea, $25 \%$ syncope/palpitations and $25 \%$ fever. All patients with deep venous thrombosis reported localized pain at the site of obstruction, $83 \%$ edema/cyanosis of the affected limb and $17 \%$ fever. The study of positive thrombophilia was the most frequent risk factor in both entities. The mean value of D-dimers was $3252 \mathrm{ug} / \mathrm{dL}$ and $2660 \mathrm{ug} / \mathrm{dL}$ in pulmonary thromboembolism and deep venous thrombosis, respectively. All patients started anticoagulation, three required intensive care, two had sequelae and one died.

Discussion: All patients had at least one risk factor, and hereditary hypercoagulability was most commonly established.

Conclusions: The increased incidence in the pediatric population described in some studies can be attributed to an increased awareness of this pathology, medical advances and increasing survival of chronic diseases. There is a lack of evidence-based recommendations identifying patients at risk of thrombosis so that decisions can be made carefully, balancing the risk and benefit in each case.

Keywords: Child; Pulmonary Embolism; Venous Thrombosis

\section{INTRODUÇÃO}

O tromboembolismo venoso (TV) inclui a trombose venosa profunda (TVP) e o tromboembolismo pulmonar (TEP). É uma patologia potencialmente devastadora com impacto significativo na qualidade de vida dos doentes pediátricos e com custos hospitalares associados. ${ }^{1}$ Com o alargamento da idade de atendimento pediátrico, a melhoria dos cuidados prestados, a maior sobrevida de doenças graves e a crescente sensibilização para eventos vasculares, o TV é cada vez mais reconhecido como um problema pediátrico significativo. ${ }^{2}$

$\mathrm{O}$ registo canadiano de TV reportou uma incidência de TEP de 0,07 por 10000 crianças e adolescentes com idades compreendidas entre um mês e 18 anos, com 5,3 por 10000 admissões hospitalares. ${ }^{3} \mathrm{Na}$ Europa, estima-se

1. Serviço de Pediatria. Centro Hospitalar Tondela-Viseu. Viseu. Portugal.

$\triangle$ Autor correspondente: Joana Verdelho Andrade. joanaverdelhoandrade@gmail.com

Recebido: 03 de setembro de 2017 - Aceite: 02 de julho de 2018 | Copyright @ O Ordem dos Médicos 2018 
uma incidência de 0,07 a 0,14 por 10000 crianças e uma taxa de mortalidade aproximada de $2 \%{ }^{4}$

A fisiopatologia do TV é explicada pela tríade de Virchow, que inclui a estase venosa, a lesão endotelial e estado de hipercoagulabilidade. A etiologia é multifatorial, existindo na maioria dos casos algum fator de risco subjacente; na faixa etária pediátrica, apenas $5 \%-10 \%$ dos casos são descritos como idiopáticos, comparativamente com $60 \%$ em adultos. ${ }^{4-7}$

O TV pode ser classificado de várias formas, entre elas: episódio inaugural ou recorrente, sintomático versus assintomático, agudo versus crónico, veno-oclusivo versus não oclusivo, idiopático ou associado a fatores de risco. Quanto aos fatores de risco protrombóticos incluem-se a administração exógena de estrogénios, presença de cateter venoso central, reduzida mobilidade e presença de trombofilia (como anticorpos antifosfolipídicos transitórios ou persistentes, deficiências adquiridas ou congénitas de anticoagulantes, mutações do fator $V$ de Leiden ou da protrombina G20210A). A introdução do termo trombofilia descreve uma predisposição aumentada, usualmente genética, para a ocorrência de fenómenos trombóticos. ${ }^{2}$

Foi demonstrado que fatores de risco agudos contribuem para o desenvolvimento de TV, tanto isoladamente como em contexto de uma doença crónica; o trauma e estados pós-cirúrgicos são fatores de risco frequentes ao seu desenvolvimento em crianças e adolescentes sem doença crónica. Outros fatores de risco comuns incluem doenças infecciosas, o uso de contraceptivos orais, a utilização prolongada de alimentação parentérica e outras condições inflamatórias. ${ }^{7,8}$ No registo Canadiano de trombose pediátrica, as causas mais prevalentes de TV foram a presença de cateter venoso central, doença maligna subjacente e status pós-cirurgia cardíaca por cardiopatia congénita, ${ }^{5}$ enquanto que um estudo coorte recente dos Estado Unidos da América, revelou como causas mais frequentes as doenças infecto-contagiosas e a presença de cateter venoso central. ${ }^{6}$

Relativamente ao estado de hipercoagulabilidade, os fatores de risco sanguíneos no TV pediátrico incluem doenças trombofílicas hereditárias e adquiridas e marcadores de ativação da coagulação. Os traços trombofílicos congénitos (exemplo, mutação no fator $\mathrm{V}$ Leiden e protrombina G20210A) são comuns na população caucasiana, com prevalência aproximada de $5 \%$ e $2 \%$ respetivamente. Exemplos comuns de trombofilia adquirida em crianças incluem o aumento da atividade do fator VIII em infeções significativas e estados inflamatórios, deficiências de anticoagulantes por consumo e coagulação intravascular disseminada na sepsis bacteriana, produção de anticorpos inibidores nas infeções agudas víricas e desenvolvimento parainfeccioso de anticorpos antifosfolipídicos. ${ }^{2}$

De facto, estima-se que mais de $60 \%$ da predisposição para trombose seja atribuível a componentes genéticos..$^{9,10}$ Os doentes com trombofilia geralmente apresentam o seu primeiro evento trombótico antes dos 25 anos e as possibilidades de recorrência aumentam com a idade e com a associação de outros fatores de risco. O risco de trombose devido a este estado é baixo antes dos 15 anos, aumentando a partir desta idade $2 \%$ a $4 \%$ ao ano. ${ }^{12}$

Para apreciar a magnitude do TV associado a fatores de risco por traços trombofílicos congénitos ou genéticos, o risco estimado baseado em populações com TV derivado de estudos em adultos é apresentado na Tabela $1 .^{2}$

O grau de suspeição de TV agudo deve ter em conta a presença de fatores de risco trombóticos clínicos e a história familiar de TV precoce ou outras doenças vasculares, bem como antecedentes de traços trombofílicos conhecidos e sinais/sintomas clínicos. Estes últimos dependem da localização anatómica e do sistema/órgão envolvido, sendo influenciado pela caraterística veno-oclusiva e cronicidade. A manifestação clássica da TVP de uma extremidade é edema doloroso unilateral de um membro. No caso de atingimento da veia cava superior, os sinais e sintomas podem incluir edema cervical e facial e cefaleia. O TEP manifesta-se classicamente por dispneia aguda inexplicável com dor pleurítica associada, mas em idade pediátrica, pode ser assintomático ou produzir sintomas ligeiros, especialmente quando envolve segmentos limitados das artérias pulmonares. No caso de atingimento da veia renal, a apresentação clínica típica é hematúria e trombocitopenia, podendo estar associado a massa abdominal e oligoanúria (se bilateral). A TV crónica pode ser detetada incidentalmente em exames imagiológicos pedidos por outros motivos, como ocorre na trombose dos seios venosos cerebrais assintomática ou alternativamente apresentar-se com sinais e sintomas de obstrução venosa crónica ou síndrome pós-trombótico secundário a trombose venosa das extremidades, incluindo dor de membro e edema, veias colaterais superficiais dilatadas, dermatite de estase venosa ou ulceração da pele. ${ }^{2}$

A avaliação laboratorial do TV pediátrico inclui hemograma, estudo da coagulação, avaliação de trombofilia e teste de beta-hCG em adolescentes pós-menarca. A Tabela 2 sumariza o painel de traços trombofílicos e marcadores dos fatores de risco de TV nos estudos pediátricos e recomendados pela Scientific and Standardization Committee Subcommitee em hemostase perinatal e pediátrica da

Tabela 1 - Risco estimado de TV em traços trombofílicos selecionados

\begin{tabular}{lc}
\hline Trombofilia & Risco estimado de TV (xBaseline) \\
\hline Hiperhomocisteinemia & 2,5 \\
Mutação da protrombina 20210, heterozigotia & 3 \\
Mutação do fator V de Leiden, heterozigotia & $2-7$ \\
Contraceptivo oral + Mutação do fator V de Leiden, heterozigotia & 35 \\
Mutação do fator V de Leiden, homozigotia & 80 \\
\hline
\end{tabular}


Tabela 2 - Condições trombofílicas associadas a TV agudo na idade pediátrica

\begin{tabular}{l} 
Trombofilia \\
\hline Genético \\
Polimorfismo do fator V de Leiden \\
Polimorfismo da protrombina 20210 \\
Elevação da concentração de lipoproteína (a) plasmática \\
Adquirido ou genético \\
Deficiência de antitrombina \\
Deficiência de proteína C \\
Deficiência de proteína S \\
Elevação da atividade do fator VIII plasmático \\
Hiperhomocisteinemia \\
Anticorpos antifosfolipídico \\
Coagulação intravascular disseminada \\
Resistência à proteína C ativada
\end{tabular}

International Society on Thrombosis and Haemostasis para a avaliação laboratorial do diagnóstico de TV aguda na criança e adolescente..$^{2,13}$

A suspeita de TVP dos membros distais ou proximais pode ser confirmada através da ecografia com Doppler. Quando se trata de envolvimento de veias do períneo e abdómen, a ressonância magnética ou tomografia computorizada é frequentemente necessária. Para confirmação de TEP, é habitualmente utilizado a tomografia computorizada espiral ou o cintigrama pulmonar de ventilação/perfusão. ${ }^{2,13}$

Em termos terapêuticos, os agentes antitrombóticos convencionais mais frequentemente utilizados são a heparina e a varfarina, sendo os níveis alvo de anticoagulação baseados em recomendações pediátricas recentes. Estes agentes atenuam a hipercoagulabilidade inerente a esta patologia, diminuindo o risco de progressão do trombo e embolismo, confiando em mecanismos fibrinolíticos intrínsecos que permitam dissolver progressivamente o trombo. ${ }^{14}$

Embora aproximadamente metade dos eventos venosos trombóticos tratados com anticoagulação tradicional durante 3-6 meses se resolva, o risco de síndrome pós-trombótico persiste, provavelmente devido a obstrução venosa completa e alterações inflamatórias locais. ${ }^{15-17}$ Enquanto a terapêutica anticoagulante atenua a coagulação em si, a terapêutica trombolítica aumenta a fibrinólise ao catalisar a ativação de plasminogénio em plasmina. ${ }^{2}$ Apesar do seu uso cada vez mais frequente, as indicações para a terapêutica trombolítica em crianças não estão bem estabelecidas. A American College of Chest Physicians recomenda o seu uso em tromboembolismo venoso com risco de vida, de órgão ou de membro, não devendo ser utilizada de forma usual. ${ }^{14}$ A American Heart Association considera esta terapêutica em idade pediátrica quando os benefícios suplantam os riscos. ${ }^{18}$

A American College of Chest Physicians sugere ainda trombectomia em crianças com tromboembolismo com risco de vida. Nas crianças com mais de $10 \mathrm{~kg}$ de peso, tromboembolismo dos membros inferiores e contraindicação para terapêutica anticoagulante sugere a colocação de filtro de veia cava inferior. ${ }^{14}$

As complicações do TV podem ser agudas se resultam das intervenções antitrombóticas ou do evento trombótico em si. Das complicações a longo prazo refere-se TV recorrente, hipertensão arterial crónica e doença renal crónica no caso de envolvimento das veias renais, hemorragia de varizes na trombose da veia porta, síndrome da veia cava superior crónica e desenvolvimento de síndrome pós-trombótico. ${ }^{16}$

Os objetivos deste trabalho consistiram em rever a epidemiologia, a apresentação clínica, os resultados de exames complementares de diagnóstico e prognóstico de doentes pediátricos com tromboembolismo pulmonar e trombose venosa profunda. Para além disso, foi também âmbito deste trabalho descrever a ocorrência de potenciais fatores de risco conhecidos desta patologia na nossa amostra de doentes pediátricos.

\section{MATERIAL E MÉTODOS}

Realizou-se um estudo retrospetivo, descritivo e analítico, onde foram incluídos todos os doentes com TV, incluindo o TEP e a TVP, com idade inferior a 18 anos, internados num hospital de nível II, durante um período de 15 anos.

Recolheram-se os dados das crianças e adolescentes internados entre 1 de janeiro de 2000 e 31 de dezembro de 2014.

As variáveis demográficas estudadas foram o género, antecedentes pessoais e familiares de relevo. As variáveis relacionadas com o TV foram idade ao diagnóstico, sinais e sintomas presentes, fatores de risco associados, comorbilidades, tratamento, evolução e complicações.

$\mathrm{Na}$ avaliação diagnóstica, consideraram-se os casos de TEP confirmados radiologicamente e de TVP comprovados ecograficamente. Foi considerado obesidade, um índice de massa corporal superior ao percentil 97 nas curvas da Organização Mundial de Saúde para a idade e género. $O$ estudo foi aprovado pela Comissão de Ética para a Saúde do Centro Hospitalar Tondela-Viseu E.P.E, tendo dispensado o consentimento informado.

\section{Análise dos dados}

Os dados foram recolhidos e codificados numa matriz inserida numa aplicação informática Excel ${ }^{\circledR}$, versão 2011 (Microsoft Corporation, EUA) e com recurso ao software SPSS ${ }^{\circledR}$, versão 20 para Mac (SPSS, IL, EUA), para análise descritiva de um conjunto de variáveis para caracterizar o grupo de doentes, bem como a construção de intervalos de confiança (IC) a $95 \%$.

\section{RESULTADOS}

No período de tempo estudado, identificaram-se 11 doentes com TV que motivaram 13 internamentos: sete casos com TEP, cinco com TVP e um com as duas últimas patologias, tendo tido três episódios trombóticos, dois TEP e uma TVP. Ocorreram quatro eventos até 2007 (31\% dos 


\section{Tromboembolismo venoso}



Figura 1 - Distribuição dos doentes com TV por ano

casos) e nos anos seguintes nove (69\% dos casos) (Fig. 1 ), sendo que esta patologia correspondeu a $0,0004 \%$ dos internamentos neste período.

Constatou-se uma preponderância do género feminino (64\%). A idade média e mediana ao diagnóstico foi de 16 anos $( \pm 1,4), 95 \%$ IC 15,2 - 17,0, com um mínimo de 13 e máximo de 17 anos.

Todos os doentes tinham pelo menos um fator de risco associado, sendo que $9 \%$ tinha um fator, $37 \%$ dois, $18 \%$ três, $27 \%$ quatro e $9 \%$ cinco fatores de risco (Tabela 3 ). 0 fator de risco mais frequente nas duas entidades foi a presença de trombofilia, seguido de obesidade e utilização de contraceptivo oral.

Tabela 3 - Fatores de risco associados aos casos de TV

\begin{tabular}{ll}
\hline \multicolumn{1}{c}{ Fatores de risco } & $\mathbf{n}$ \\
\hline Trombofilia & 10 \\
\hline Mutação de MTHFR & \\
MTHFR C677T + MTHFR A1298C, dupla heterozigotia & 3 \\
MTHFR C677T, heterozigotia & 1 \\
MTHFR A1298C, heterozigotia & 1 \\
Hiperhomocisteinemia & 2 \\
Défice de antitrombina & 2 \\
Défice de proteína S & 1 \\
Mutação do fator V de Leiden, heterozigotia & 1 \\
Mutação da protrombina 20,210, heterozigotia & 1 \\
Síndrome dos anticorpos antifosfolipídicos & 1 \\
Antecedentes pessoais & \\
\hline Obesidade & 6 \\
Contraceptivo oral & 4 \\
Incapacidade motora ou intelectual severa & 3 \\
Fumador & 3 \\
Cirurgia recente & 1 \\
Insuficiência cardíaca & 1 \\
Trombose venosa profunda prévia & 1 \\
Trauma & 1 \\
\hline Viagem prolongada recente & 1 \\
\hline
\end{tabular}

Antecedentes familiares

Pai e Irmã com antecedente de trombose venosa profunda
Todos os casos com hiperhomocisteinémia possuíam mutação MTHFR C677T e MTHFR A1298C em dupla heterozigotia. Para além disso, constatou-se que duas adolescentes apresentavam alterações trombofílicas concomitantes: MTHFR C677T em heterozigotia e défice de proteína S; MTHFR C677T e MTHFR A1298C em dupla heterozigotia e défice de antitrombina.

Os três doentes com incapacidade motora ou intelectual severa corresponderam a paraplegia decorrente de acidente de viação com lesão a nível de D9, perturbação do espectro do autismo e trissomia 21.

Relativamente aos casos de TEP $(n=8)$, um dos quais com TVP concomitante, todos apresentaram toracalgia e dispneia à admissão, 25\% síncope/palpitações e 25\% febre (Fig. 2). Todos realizaram tomografia computorizada para confirmação de diagnóstico e em cinco casos verificou-se envolvimento pulmonar bilateral. O caso com TVP concomitante apresentava envolvimento das veias ilíacas e femorais.

Quanto aos casos com TVP isolada $(n=5)$, todos referiam dor no local da obstrução, $83 \%$ edema e cianose do membro afetado e $17 \%$ febre (Fig. 2). Todos realizaram ecografia com Doppler que confirmou o diagnóstico. As localizações mais frequente foram as veias ilíacas e femorais $(n=3)$ seguido das veias tibial e poplítea $(n=2)$.

Todos os casos de TV apresentavam elevação dos D-dímeros ao diagnóstico (Tabela 4).

A duração média de internamento foi de 14 dias (mínimo 1 , máximo $25,95 \%$ IC 8,8 - 18,8) e de 8 dias (mínimo 3 , máximo $24,95 \%$ IC 3,4 - 16,4), no TEP e na TVP, respetivamente. Três dos oito casos de TEP foram transferidos para o hospital de nível III, por necessidade de cuidados intensivos pediátricos.

Todos realizaram tratamento com anticoagulante com heparina de baixo peso molecular como tratamento inicial. Dois casos com TEP necessitaram de terapêutica com agentes trombóticos ativadores do plasminogénio tecidular, apresentando um deles insuficiência ventricular direita. Após terapêutica inicial com heparina de baixo peso molecular, entre 5 a 10 dias, iniciaram de forma concomitante e posteriormente isoladamente a varfarina. Não houve necessidade de embolectomia, nem de colocação de filtros da veia cava inferior. A duração de tratamento com varfarina 


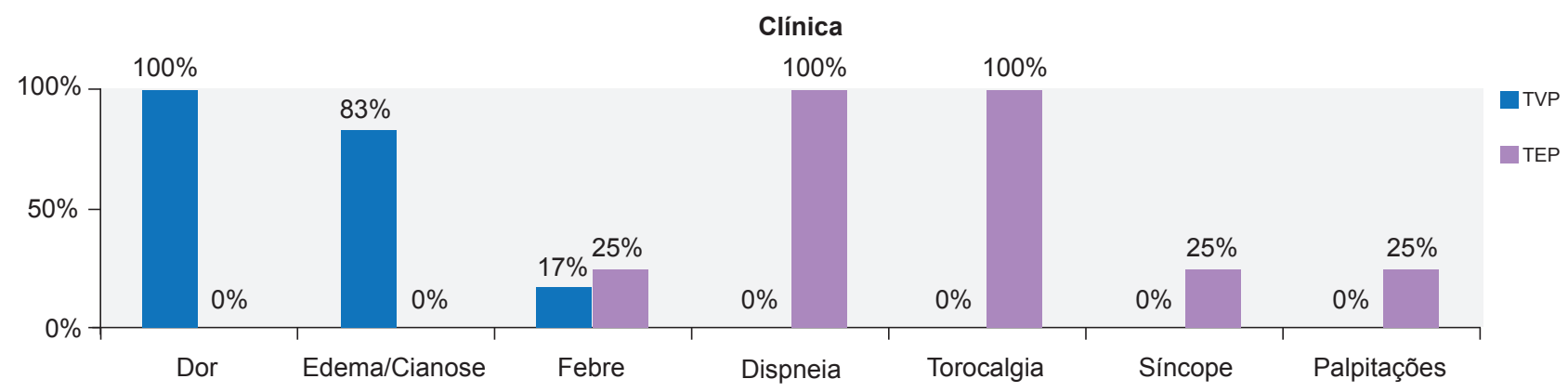

Figura 2 - Distribuição dos doentes com TVP e TEP quanto aos sinais e sintomas

Tabela 4 - Exames complementares de diagnóstico realizados nos casos com TEP e TVP

\begin{tabular}{lcc}
\hline \multicolumn{1}{c}{ Exames complementares de diagnóstico } & TEP & TVP \\
\hline Leucócitos $>15,0 \times 10^{9} / \mathrm{L}$ & $12 \%$ & $20 \%$ \\
Plaquetas $<150.000 / \mathrm{LL}$ & $0 \%$ & $0 \%$ \\
Prolongamento do tempo de tromboplastina parcialmente ativada & $13 \%$ & $17 \%$ \\
Proteína C reativa $>4,0 \mathrm{mg} / \mathrm{dL}$ & $88 \%$ & $80 \%$ \\
& Média 3252 & Média 2660 \\
& Mínimo 661 & Mínimo 862 \\
D-dímeros $(\mathrm{ug} / \mathrm{mL})$ & Máximo 14239 & Máximo 5102 \\
& $95 \%$ IC 1303- 7048 & $95 \%$ IC $862-5102$ \\
\hline
\end{tabular}

foi de 3-6 meses nos casos em que o fator de risco foi resolvido. Nos casos de risco crónico, a duração da terapêutica prolongou-se até 12 meses.

Verificou-se um óbito em fase aguda, um caso de TEP com recorrência de eventos trombóticos por má adesão à terapêutica instituída e um caso que resultou em síndrome pós-trombótico nos casos de TVP.

\section{DISCUSSÃO}

No nosso estudo, todos os casos ocorreram em adolescentes, não se detetando nenhum caso na infância. Sabe-se que existem dois picos na incidência em idade pediátrica: lactentes e adolescentes. Ao longo das últimas décadas, tem-se assistido a um progresso significativo no conhecimento dos mecanismos fisiopatológicos envolvidos na TV. ${ }^{9}$ Nos diversos estudos, a maioria das crianças tem alguma condição clínica inerente, sendo que a presença de cateter venoso central é a causa mais frequentemente descrita. ${ }^{10}$ No nosso trabalho, não se detetou nenhum caso na infância. Todos os nossos adolescentes tinham pelo menos um fator de risco conhecido para TV. O avanço mais significativo no conhecimento da fisiopatologia da TV foi a confirmação de que existem condições de hipercoagulabilidade herdadas presentes numa grande percentagem de doentes com TVP e TEP, tendo sido o fator de risco mais frequentemente encontrado nos nossos adolescentes.

Das sete adolescentes com TV, quatro tomavam contraceptivo oral e destas três tinham trombofilia desconhecida até ao evento. O risco de TV relacionado com o uso de compostos hormonais está relacionado com a dose de estrogénio, o tipo de progestativo, idade, história familiar e a presença de trombofilia. Em 2010, o Centers for Disease
Control and Prevention apresentou as recomendações do Colégio Americano de Obstetrícia e Ginecologia, que permitia considerar-se contraceptivos orais em doentes com TV atribuível a fator de risco reversível ou sob anticoagulante. Por outro lado, caso a TV prévia tenha ocorrido sob contraceptivo oral ou em grávida, a terapêutica hormonal seria uma contraindicação absoluta. Apesar da atenção dada à trombofilia, nenhuma das recomendações sugere rastreio universal, dado que o custo será elevado. Para além de que o rastreio pode produzir uma falsa tranquilidade, ansiedade, acompanhamento exagerado, rastreio familiar adicional e significativa e desnecessária despesa, bem como, quando negativo, uma falsa tranquilidade quanto à ocorrência de eventos tromboembólicos. ${ }^{19}$

O presente estudo também identificou uma forte prevalência de obesidade na nossa amostra (55\%), o que está de acordo com a literatura. ${ }^{20}$ Estudos portugueses demonstraram uma prevalência de excesso de peso/obesidade de cerca de $32 \%$ até aos 36 meses de idade ${ }^{21}$ e $35 \%$ - 37\% em crianças e adolescentes. ${ }^{22,23}$ Como a obesidade é um fator de risco conhecido de TV e tem vindo a aumentar a nível mundial a um ritmo alarmante, este facto pode contribuir para um aumento da incidência de fenómenos trombóticos na população pediátrica.

O caso com TEP e TVP concomitante apresentava vários fatores de risco que incluíam cirurgia recente, antecedentes prévios de TVP, obesidade e trombofilia, para além de não compliance à medicação.

Existem recomendações que avaliam e classificam o risco de TV e que abordam de forma estratificada a sua profilaxia, pelo que é importante sensibilizar os profissionais de saúde para a diferenciação desse mesmo risco, 
embora ainda haja pouca evidência pediátrica que suporte estratégias profiláticas específicas. ${ }^{24,25}$

Relativamente ao tratamento instituído, o nosso trabaIho denota que foram seguidas as recomendações internacionais. Não houve necessidade de embolectomia ou de colocação de filtros da veia cava inferior em nenhum caso.

O TEP é raro, mas potencialmente fatal em idade pediátrica. Quer a nível internacional, quer no nosso país, carecem estudos multicêntricos da evolução de TEP em idade pediátrica. O registo Canadiano e Holandês reportam uma mortalidade global de TV de cerca de $10 \%$, e particularmente nos casos com TEP, no primeiro registo, dois óbitos por vinte e duas crianças e, no segundo, um óbito por cada dez. ${ }^{3,4}$ No nosso trabalho, tivemos um óbito em 11 adolescentes, o que é semelhante aos dados internacionais.

Existem algumas limitações do nosso estudo. $O$ facto de os nossos doentes pertencerem a apenas uma instituição e a amostra ser pequena, não permite extrapolar resultados. Todos os dados foram recolhidos retrospectivamente dos processos clínicos dos doentes e dos dados médicos informatizados, pelo que estudos futuros poderiam incluir uma análise multicêntrica e com grupo controlo com rastreio estandardizado da trombofilia de todos os doentes.

\section{CONCLUSÃO}

O TV ocorre em idade pediátrica e é uma patologia rara. $\mathrm{O}$ aumento da incidência descrito em alguns trabalhos pode ser atribuído a uma crescente sensibilização desta patologia, aos progressos médicos e tecnológicos e a um aumento da sobrevida de doenças crónicas. No entanto, a incidência relatada está provavelmente subestimada pois o TEP é frequentemente silencioso ou apresenta-se com sintomas inespecíficos. Este estudo enfatiza os fatores de risco que devem aumentar o índice de suspeição.

Para além disso, as estratégias diagnósticas e terapêuticas utilizadas em crianças são extrapoladas de estudos em adultos, pelo que escasseiam recomendações baseadas na evidência de forma a identificar os doentes pediátricos com risco de trombose, para que as decisões possam ser tomadas de forma cuidadosa, equilibrando o risco e benefício em cada caso.

São necessários estudos futuros que investiguem a razão da crescente incidência de TV, podendo ser resultante da maior sobrevida de crianças com doença crónica, do maior número de doentes pediátricos com cateteres venosos centrais e da maior prevalência de obesidade. É importante estudar a evolução do TV pediátrico, incluindo a mortalidade, a recorrência de eventos trombóticos e a função pulmonar. Finalmente, para melhorar a assistência a estes doentes são essenciais e necessários trabalhos multicêntricos que avaliem os fatores de risco e a apresentação clínica de forma a desenvolver recomendações fiáveis e reprodutíveis que permitam determinar a probabilidade de TV em idade pediátrica.

\section{OBSERVAÇÕES}

Trabalho apresentado como comunicação oral no $16^{\circ}$ Congresso Nacional de Pediatria, que decorreu de 22 a 24 de Outubro de 2015, em Albufeira.

\section{PROTECÇÃO DE PESSOAS E ANIMAIS}

Os autores declaram que os procedimentos seguidos estavam de acordo com os regulamentos estabelecidos pelos responsáveis da Comissão de Investigação Clínica e Ética e de acordo com a Declaração de Helsínquia da Associação Médica Mundial.

\section{CONFIDENCIALIDADE DOS DADOS}

Os autores declaram ter seguido os protocolos do seu centro de trabalho acerca da publicação de dados.

\section{CONFLITO DE INTERESSES}

Os autores declaram não ter nenhum conflito de interesses na realização do presente artigo.

\section{FONTES DE FINANCIAMENTO}

Não existiram fontes externas de financiamento para a realização deste artigo.

\section{REFERÊNCIAS}

1. Candrilli SD, Balkrishnan R, O'Brien SH. Effect of injury severity on the incidence and utilization related outcomes of venous thromboembolism in pediatric trauma inpatients. Pediatr Crit Care Med. 2009;10:554-7.

2. Goldenberg NA, Bernard TJ. Venous Thromboembolism in Children. Hematol Oncol Clin N Am. 2010;24:151-66.

3. Andrew M, David M, Adams M, Ali K, Anderson R, Barnard D et al. Venous thromboembolic complications (VTE) in children: first analyses of the Canadian Registry of VTE. Blood. 1994;83:1251-7.

4. van Ommen $\mathrm{CH}$, Heijboer $\mathrm{H}$, Buller $\mathrm{HR}$, Hirasing RA, Heijmans $\mathrm{HS}$, Peters $\mathrm{M}$ Venous thromboembolism in childhood: a prospective twoyear registry in the Netherlands. J Pediatr. 2001;139:676-81.

5. Monagle P, Adams M, Mahoney M, Ali K, Barnard D, Bernstein M, et al. Outcome of pediatric thromboembolic disease: a report from the Canadian Childhood Thrombophilia Registry. Pediatr Res. 2000;47:7636.

6. Goldenberg NA, Knapp-Clevenger R, Manco-Johnson MJ, Mountain States Regional Thrombophilia Group. Elevated plasma factor VIII and D-dimer levels as predictors of poor outcomes of thrombosis in children. N Engl J Med. 2004;351:1081-8

7. Setty BA, O'Brien SH, Kerlin BA. Pediatric Venous Thromboembolism in the United States: a tertiary care complication of chronic diseases. Pediatr Blood Cancer. 2012;59:258-64.

8. Takemoto CM, Sohi S, Desai K, Bharaj R, Khanna A, McFarland S et al. Hospital-associated venous thromboembolism in children: incidence and clinical characteristics. J Pediatr. 2014;164:332-8.

9. Rosendaal FR. Venous thrombosis: a multicausal disease. Lancet. 1999;353:1167-73.

10. Fedeman DG, Kirsner RS. An update on hypercoagulable disorders. Arch Intern Med. 2001;161:1051-6.

11. Franco RS, Reitsman PH. Genetic risk factors of venous thrombosis. Hum Genet. 2001;109:369-84.

12. Laffan M, Tuddenham E. Assessing thrombotic risk. BMJ. 1998:317:5203.

13. Manco-Johnson MJ, Grabowski EF, Hellgreen M, Kemahli AS, Massicotte MP, Muntean W, et al. Laboratory testing for thrombophilia in pediatric patients. On behalf of the Subcommittee for Perinatal and Pediatric Thrombosis of the Scientific and Standardization Committee of the International Society on Threombosis and Haemostasis (ISTH). 
Thromb Hae Most. 2002;88:155-6.

14. Monagle P, Chan AKC, Goldenberg NA, Ichord RN, Journeycake JM, Nowak-Göttl U, Vesely SK. Antithrombotic therapy in neonates and children: Antithrombotic Therapy and Prevention of Thrombosis, 9th ed: American College of Chest Physicians Evidence-Based Clinical Practice Guidelines. Chest. 2012;141:e737S-e801.

15. Raffini L. Thrombolysis for intravascular thrombosis in neonates and children. Curr Opin Pediatr. 2009;21:9-14.

16. Goldenberg NA. Long-term outcomes of venous thrombosis in children. Curr Opin Hematol. 2005;12:370-6.

17. Goldenberg NA, Donadini MP, Kahn SR, Neil A, Crowther M, Kenet $\mathrm{G}$, et al. Post-thrombotic syndrome in children: a systematic review of frequency of occurrence, validity of outcome measures, and prognostic factors. Haematologica. 2010;95:1952-9.

18. Jaff MR, McMurtry MS, Archer SL, Cushman M, Goldenberg N, Goldhaberet SZ, et al. Management of massive and submassive pulmonary embolism, iliofemoral deep vein thrombosis, and chronic thromboembolic pulmonary hypertension: a scientific statement from the American Heart Association. Circulation. 2011;123:1788-830.

19. Centers for Disease Control and Prevention (CDC). U S. Medical Eligibility Criteria for Contraceptive Use, 2010. MMWR Recomm Rep. 2010;59:1-86.

20. Agha BS, Sturm JJ, Simon HK, MD, Hirsh DA. Pulmonary embolism in the pediatric emergency department. pediatrics. 2013;132:663-7.

21. Vale S, Trost SG, Rêgo C, Abreu S, Mota J. Physical activity, obesity status, and blood pressure in preschool children. J Pediatr. 2015;167:98102.

22. Camarinha B, Graça P, Nogueira PJ. A prevalência de pré-obesidade/ obesidade nas crianças do ensino pré-escolar e escolar na autarquia de Vila Nova de Gaia, Portugal. Acta Med Port. 2016;29:31-40.

23. Viveiro $C$, Brito S, Moleiro P. Sobrepeso e obesidade pediátrica: a realidade portuguesa. Rev Port Saúde Publica. 2016;34:30-7.

24. Meier KA, Clark E, Tarango C, Chima RS, Shaughnessy E. Venous thromboembolism in hospitalized adolescents: an approach to risk assessment and prophylaxis. Hosp Pediatr. 2015;5:44-51.

25. Mahajerin A, Webber EC, Morris J, Taylor K, Saysana M. Development and implementation results of a venous thromboembolism prophylaxis guideline in a tertiary care pediatric hospital. Hosp Pediatr. 2015;5:6306.

26. van Ommen $\mathrm{CH}$, Peters $\mathrm{M}$. Acute pulmonary thromboembolism in childhood. Thromb Res. 2006;118:13-25.

27. Streif W, Andrew ME. Venous thromboembolic events in pediatric patients: Diagnosis and Management. Hematol Oncol Clin North Am. 1998;12:1283-312

28. Van Ommen $\mathrm{CH}$, Peters $\mathrm{M}$. Venous thromboembolic disease in childhood. Semin Thromb Hemost. 2003;29:391-404. 